\title{
Cancer/testis antigen SSX2 enhances invasiveness in MCF-7 cells by repressing ER $\alpha$ signaling
}

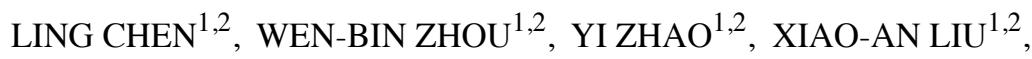 \\ QIANG DING ${ }^{1,2}$, XIAO-MING ZHA ${ }^{1,2}$ and SHUI WANG ${ }^{1,2}$ \\ ${ }^{1}$ Department of Breast Surgery, The First Affiliated Hospital with Nanjing Medical University; ${ }^{2}$ State Key \\ Laboratory of Reproductive Medicine, Department of Histology and Embryology, \\ Nanjing Medical University, Nanjing, P.R. China
}

Received November 13, 2011; Accepted January 5, 2012

DOI: 10.3892/ijo.2012.1369

\begin{abstract}
Cancer/testis antigen (CTA) SSX2, which is silent in most normal adult tissues and expressed in various malignant tumors, has been identified for decades. Expression of SSX in tumors has been associated with advanced stages and worse patient prognosis. However, little is known about its role in breast cancer. The SSX2 expression plasmid constructed was stably transfected into the breast cancer cell line MCF-7. The influence of SSX2 on MCF-7 cells was assessed using MTT assay, flow cytometry, transwell invasion assay and in vivo tumorigenicity assay. A comparative proteomic approach was performed to identify and clarify the underlying molecular mechanisms. SSX2 expression was more pronounced in ER $\alpha$-negative breast cancer cells compared with the positive ones. Overexpression of SSX2 induced cell growth and prompted cell invasion. Both $\mathrm{ER} \alpha$ and E-cadherin expression were suppressed in the SSX2 overexpressing MCF-7 cells. Eleven known proteins were identified with significant differential expression. Among these, five were decreased, while other six were increased in the SSX2 overexpressing MCF-7 cells. These results suggested SSX2 may enhance invasiveness in $\mathrm{MCF}-7$ cells both in vivo and in vitro. The regulation of ER $\alpha$ signaling by target proteins of SSX2 may explain the metastatic potential of breast cancer cells.
\end{abstract}

\section{Introduction}

Breast cancer is the most common malignancy in females worldwide. Breast cancer alone is expected to account for $28 \%$ $(207,090)$ of all new cancer cases among American women in 2010 (1). Due to the progress of early diagnosis and systemic therapies including chemotherapy and endocrine therapy as well as targeted therapy, patients suffering from breast cancer have a better prognosis of long-term survival than ever before. However,

Correspondence to: Dr Shui Wang, Department of Breast Surgery, The First Affiliated Hospital with Nanjing Medical University, 300 Guangzhou Road, 210029 Nanjing, P.R. China

E-mail:ws0801@hotmail.com

Key words: breast cancer, cancer/testis antigen, CTA, HOM-MEL-40/ SSX2, apoptosis, invasion the disease is still responsible for the second major cause of cancer-related mortality. An emerging improvement to eradicate cancer may be brought by cancer vaccine-based immunotherapy which should be efficient and low-toxic for patients (2). Hence, detection and identification of tumor associated antigen is of urgent need.

Cancer/testis antigens (CTAs) are a unique group of tumor antigens with normal expression restricted in the testis and in various cancers but not in adult somatic tissues (3). In some cancers, the expression of CTAs impacts a poorer prognostic future (4-8). These tissue-restricted proteins can induce spontaneous immune responses when aberrantly produced in tumors as there is no or weak central tolerance against proteins restricted to immunoprivileged sites (9). Up to now, only a few CTAs have been shown to elicit both humoral and cell-mediated immune responses in human, including SSX, MAGE-A, and NY-ESO-1 (10). MAGE-A and NY-ESO-1 are expressed in a substantial proportion of triple-negative breast cancers (11). It is also reported that SSX2 is found in breast cancer patients. These CTAs are suggested to be breast cancer antigens (12). MAGE-A3 and NY-ESO-1 as tumor targets are currently evaluated in clinical trials $(13,14)$. However, study on SSX protein family is less advanced.

The SSX genes, located on the X chromosome, encode a family of at least ten highly homologous nuclear proteins (15). Expression of SSX proteins in tumor tissues has been associated with advanced stages and worse patient prognosis $(16,17)$. The SSX2 gene codes for the cancer/testis antigen HOM-MEL-40 which was found to be immunologically recognized in the sera of some melanoma patients. It was expressed in approximately $20 \%$ of breast cancer samples but not in normal tissues except for testes (18). Like other members of this family, SSX2 has Krüppel-associated box (KRAB) domain and consensual sequences for $\mathrm{N}$-glycosylation and tyrosine phosphorylation. In addition, the conserved C-terminus of SSX2 named as SSXRD (SSX repression domain) is associated with transcriptional repressor regulation $(19,20)$. The SSX KRAB domain interacts with the RAB3IP (RAB3A interacting protein) and SSX2IP (SSX2 interacting protein), while the SSXRD domain interacts with LHX4 (LIM homeobox 4) and PcG protein $(21,22)$. Current knowledge of these SSX2-interacting proteins is related to cellular growth, apoptosis and adhesion, but little is known about 
the function of SSX2 in breast cancer. Interestingly, the expression of SSX2 in malignant cells can be induced or augmented by the DNA methylation inhibitor 5-aza-2'-deoxycytidine (5-DAC) (16). It is indicated that epigenetic molecular mechanisms in regulation of SSX2 expression could be explored as a molecular strategy in the treatment of breast cancer. Given the potential target for breast cancer vaccine-based immunotherapy, it is therefore important to elucidate the roles of SSX2 protein in breast cancer cells. With this in mind, we constructed a breast cancer cell line over-expressing SSX2 protein to investigate its effects on breast cancer.

\section{Materials and methods}

Cells, reagents, and animals. Breast cancer cell lines MCF-7, MDA-MB-231, MDA-MB-468, HCC1937, SUM1315, T47D, SK-BR-3, ZR-75-1, MCF10A, were purchased from American Type Culture Collection (Manassas, VA, USA) and cultured in complete medium. pEGFP-C2 vector (BD Bioscience Clontech) was transfected by FuGENE ${ }^{\mathrm{TM}} 6$ (Roche Applied Science, USA). Restriction enzymes (EcoRI/BamHI) were from New England Biolabs (USA). Antibody toward SSX2 was from Santa Cruz Biotechnology, Inc. Antibody to $\beta$-actin was from Sigma (St. Louis, MO, USA). Antibody to E-cadherin, vimentin was from Abcam (Cambridge, UK). Antibody to ER $\alpha$ was from Cell Signaling Technology, Inc. (Beverly, MA). Goat anti-mouse IgG-HRP and rabbit anti-goat IgG-HRP were from Santa Cruz. Balb/c null mice were from SLAC Laboratory Animal (Shanghai, China).

Vector construction. Wild-type SSX2 ORF was isolated by PCR from human testis cDNA (forward primer is 5'-GGGGAATTC ATGAACGGAGACGACGCCTT-3' and reverse primer is 5'-CGCGGATCCTCACCAGCTGTTTTCTCTCA-3'). The full length of ORF is $671 \mathrm{bp}$. The fragment was cloned into pEGFP-C2 via an EcoRI/BamHI site.

Gene transfection. MCF-7 cells were transfected with pEGFPC2-SSX2 vectors using FuGENE 6 based on a transfection reagent: DNA ratio of 3:1 according to the instruction manual. Transfection with pEGFP-C2 empty vector was used as control. After antibiotic selection ( $800 \mu \mathrm{g} / \mathrm{ml} \mathrm{G} 418$; Sigma-Aldrich), transfectants were pooled to avoid the effects of clonal selection and were expanded in $400 \mu \mathrm{g} / \mathrm{ml} \mathrm{G} 418$. Western blot analysis was performed to detect the SSX2 expression.

$R T-P C R$. Total RNA from cells or tissue samples was extracted using TRIzol (Invitrogen, Carlsbad, CA, USA). The RNA was transcribed using the One Step RNA PCR Kit (AMV) from Takara Biotechnology. Specific primers for $\beta$-actin (forward, 5'-CTCCATCCTGGCCTCGCTTGT-3'; reverse, 5'-GCTG TCACCTTCACCGTTCC-3'), human SSX2 (forward, 5'-GTGC TCAAATACCAGAGAAGATC-3'; reverse, 5'-TTTTGGGTCC AGATCTCTCGTG-3') were from Invitrogen Co. (Shanghai, China).

Western blot assay. The protein levels of SSX2, E-cadherin, vimentin, ER $\alpha$ in SSX2 over-expressing MCF-7 and the controls were analyzed by Western blotting using previously described methods. Primary antibodies included mouse anti-E-cadherin
1:1000, mouse anti-vimentin 1:500 (clone V9), mouse anti- $\beta$ actin 1:10000 (clone AC-15). Secondary antibodies included goat anti-mouse IgG-HRP 1:1000) and rabbit anti-goat IgG-HRP 1:1000.

Growth curve by MTT assay. Cells $\left(2 \times 10^{4}\right)$ were grown in microtiter plates in a final volume of $180 \mu \mathrm{l}$ complete medium per well in $37^{\circ} \mathrm{C}, 5 \% \mathrm{CO}_{2}$. The growth assay was performed over a period of 6 days. After the incubation period, $20 \mu 1$ of the MTT labeling reagent $(0.5 \mathrm{mg} / \mathrm{ml})$ was added to each well. The cells were analyzed by enzyme-labelled meter. The absorption was measured at $570 \mathrm{~nm}$.

Apoptosis detection by flow cytometry. SSX2 over-expressing MCF-7 and the control cells were collected for apoptosis detection. The detection of apoptosis was performed according to the instruction manual of the FITC Annexin V Apoptosis Detection Kit I (BD Biosciences, San Diego, CA, USA).

Transwell invasion assay. For the transwell-based migration assay, the upper compartments of filter membranes were coated with $1 \mathrm{mg} / \mathrm{ml}$ matrigel at $4^{\circ} \mathrm{C}$. SSX2 over-expressing MCF-7 and the control cells ( $1 \times 10^{5} /$ well) were seeded on $8-\mu \mathrm{m}$ pore size transwells (Corning, NY, USA). The complete medium was then added into the lower compartments of chamber. After incubated for $24 \mathrm{~h}$, cell numbers were evaluated in the bottom compartment of the filter membranes. All in vitro experiments were performed in triplicates and similar results were obtained.

Tumorigenicity assay in nude mice. BALB/C nude $(\mathrm{nu} / \mathrm{nu})$ female mice (4-6-weeks old, 18-22 g) were randomly divided into two groups (each containing 10 mice). The study protocol was reviewed and approved by the Institutional Animal Care and Use Committee of Nanjing Medical University. A total of $5 \times 10^{6}$ cells suspended in $0.15 \mathrm{ml}$ PBS was subcutaneously injected into the right flank of each mouse. After 8 weeks, the mice were sacrificed and the xenografts were resected, fixed in $10 \%$ buffered formalin for 6-10 $\mathrm{h}$, and processed for histological analysis.

2-DE, gel image analysis and protein identification. Proteins from the two groups were extracted and separated by 2-DE. Gels were silver stained, scanned, and analyzed using ImageMaster ${ }^{\mathrm{TM}}$ 2D platinum software (Version 5.0, GE Healthcare, San Francisco, CA, USA). The expression level was determined by the relative volume of each spot in the gel and expressed as \% $\mathrm{Vol}\left[\% \mathrm{Vol}=\left(\right.\right.$ spot volume $/ \sum$ volumes of all spots resolved in the gel)]. Three independent experiments of cells were averaged respectively, calculated and assessed with the Student's t-test using ImageMaster 2D platinum software. A spot was regarded differentially expressed if the change of average spot intensity was $>1.5$-fold and $\mathrm{P}<0.05$.

Spots with significant differences were excised. Gel pieces were denatured, alkylated, trypsin digested and analyzed by an Ultraflex II MALDI-TOF-TOF mass spectrometer (Bruker Daltonics $\mathrm{GmbH}$, Bremen, Germany) under the control of FlexControl $^{\mathrm{TM}} 2.4$ software (Bruker Daltonics $\mathrm{GmbH}$ ). MALDI-TOF spectra were recorded in the positive ion reflector mode in a mass range from 700 to $4000 \mathrm{Da}$ and the ion acceleration voltage was $25 \mathrm{kV}$. Acquired mass spectra were processed 


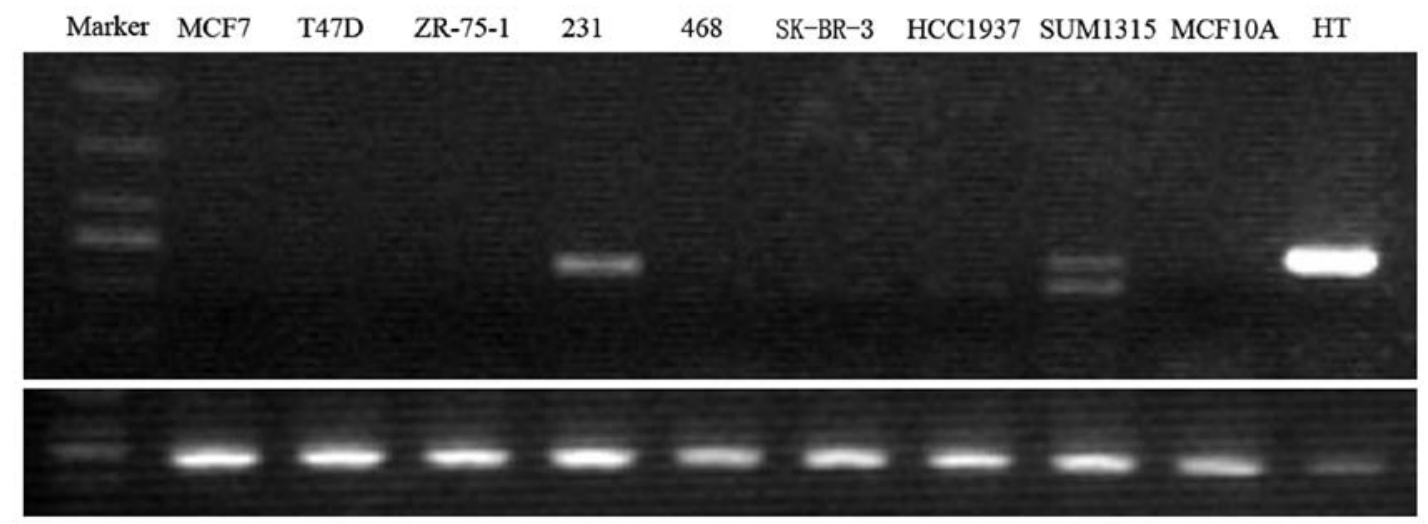

Figure 1. The differential mRNA expression of SSX2 was confirmed on the normal breast epithelial cell line MCF10A and a panel of 8 human breast cancer cell lines. The expression of SSX2 in human testis (HT) was performed as the positive control. The product length should be 435 bp. As was shown, MCF10A scarcely expressed SSX2. Breast cancer cell lines such as MDA-MB-231, HCC1937, SUM1315 had higher mRNA levels of SSX2.

using the software FlexAnalysis ${ }^{\mathrm{TM}}$ 2.4: peak detection algorithm: SNAP (sort neaten assign and place); S/N threshold: 3; quality factor threshold: 50 . The trypsic autodigestion ion picks [trypsin_(108-115), $\mathrm{MH}^{+} 842.509$, trypsin_(58-77), $\left.\mathrm{MH}^{+} 2211.104\right]$ were used as internal standards. Matrix and/or auto-proteolytic trypsin fragments, or known contaminant ions (keratins) were excluded. The resulting peptide mass lists were used to search the IPI human database 3.30 (67922 sequences, 28879402 residues) with Mascot (v2.1.03) in automated mode. The search criteria was as followed: significant protein Mowse score at $\mathrm{P}<0.05$, minimum mass accuracy $100 \mathrm{ppm}$, trypsin as enzyme, one missed cleavage site allowed, and alkylation of cysteine by carbamidomethylation as fixed modification and oxidation of methionine as variable modification. The Mascot score and expectation of the first non-homologous protein to the highest ranked hit were checked. Protein identification was confirmed by sequence information obtained from MS/ MS analysis in 'LIFT' (laser-induced forward transfer) mode. Acquired MS/MS spectra were also processed using the software FlexAnalysis 2.4 using a SNAP method set at an S/N threshold of 3.0. For MS/MS spectra searching, the spectra were used to search IPI human database 3.30 automated using Mascot (v2.1.03). The score was higher than the minimal significant $(\mathrm{P}<0.05)$ individual ion score. All significant MS/MS identifications by Mascot were manually verified for spectral quality and matching $\mathrm{y}$ and $\mathrm{b}$ ion series.

Statistical analysis. Data were analyzed by t-test for significant differences between SSX2 over-expressing MCF-7 and the control cells. All the ratios were arcsine square root transformed before t-test analysis and the least significant difference post hoc test was used to examine any significant difference between groups. The results were considered statistically significant when $\mathrm{P}<0.05$.

\section{Results}

Expression of SSX2 in breast cancer cell lines. The differential mRNA expression of SSX2 was confirmed on the normal breast epithelial cell line MCF10A and a panel of 8 human breast cancer cell lines, including both non-invasive and invasive cell lines. The expression of SSX2 in human testis was performed

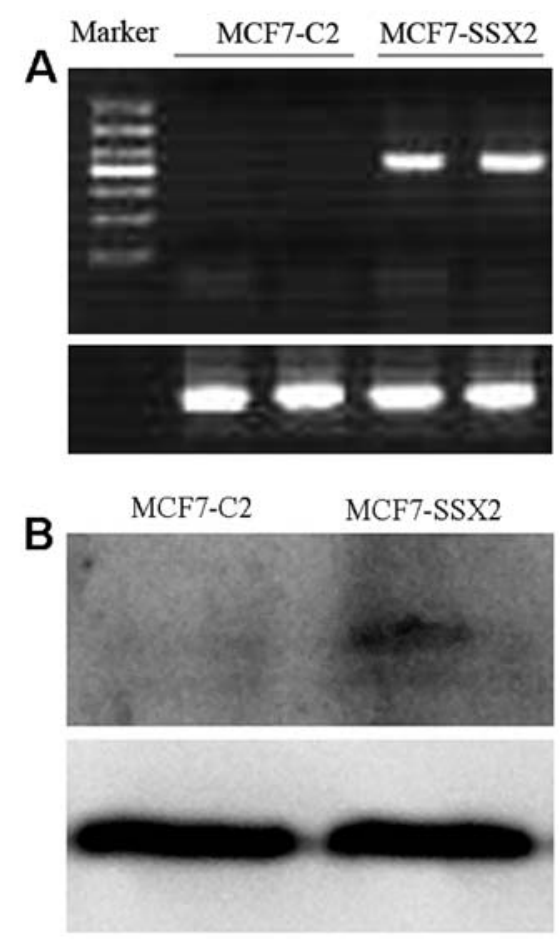

Figure 2. The constructed expression vector pEGFP-C2-SSX2 was stably transfected into MCF-7 which was SSX2-negative. Both mRNA (A) and protein level (B) were confirmed.

as the positive control. The MCF10A scarcely expressed SSX2. Interestingly, ER $\alpha$-negative breast cancer cell lines such as MDA-MB-231, HCC1937, SUM1315 have a higher mRNA level of SSX2, compared with ER $\alpha$-positive cell lines such as MCF-7, T47D, ZR-75-1 (Fig. 1). To note, cell lines with higher mRNA levels of SSX2 were more likely to be hormone receptor-negative.

SSX2 expression involves with the growth of MCF-7 cells. For revealing the function of SSX2 on cell growth, the constructed expression vector pEGFP-C2-SSX2 was stably transfected into MCF-7 which was SSX2-negative. Both mRNA and protein level were confirmed (Fig. 2). The growth curve of 


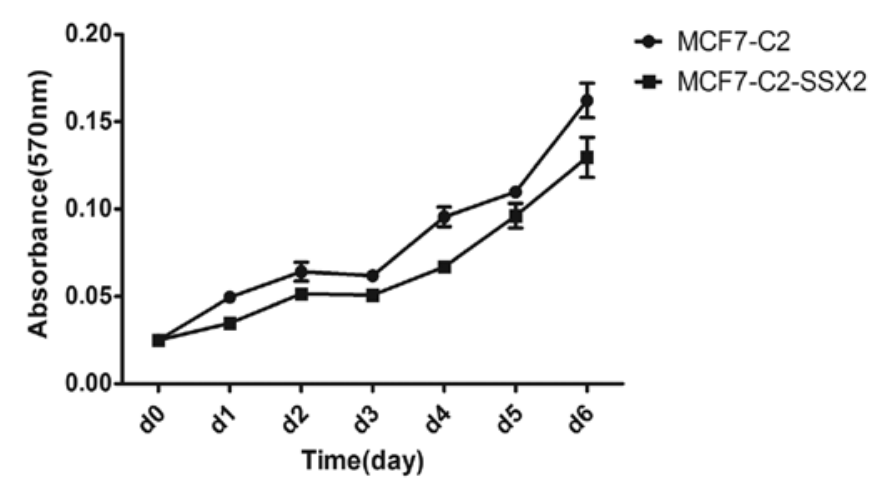

Figure 3. The growth curve was performed on a-week period. The proliferation rate of SSX2 over-expressing cells showed a markedly decrease.

SSX2 over-expressing cells showed a markedly decrease compared with the control cells by using MTT assay (Fig. 3). To observe the effects of SSX2 on breast cancer cell apoptosis, the detection of apoptosis by flow cytometry was performed. The over-expression of SSX2 promoted a significant increase in the early-stage apoptosis rate of MCF-7 (Fig. 4). We found that upregulation of SSX2 expression could induce early apoptosis, which indicated that SSX2 was a pro-apoptotic factor during programmed cell death of MCF-7. It was surmised that SSX2 protein was involved in breast cancer cell survival.

SSX2 expression enhances MCF-7 cell invasiveness. The invasiveness of SSX2 over-expressing MCF-7 and the control cells were assessed by transwell assays. In the transwell assay, the invasiveness through $8-\mu \mathrm{m}$ pore transwells was significantly $(\mathrm{P}<0.05)$ increased in SSX2 over-expressing MCF-7, compared with the controls (Fig. 5). The average number of SSX2 over- expressing MCF-7 that migrated for $24 \mathrm{~h}$ was 63 cells/site, comparing with 31 cells/site for the control cells.

Over-expression of SSX2 decreases ER $\alpha$ and E-cadherin expression in MCF-7. The expression level of protein markers relative to MCF-7 was assessed after the transfection. As is shown in Fig. 6, ER $\alpha$ expression level was markedly decreased in the SSX2 over-expressing MCF-7 compared with the control cells. Interestingly, the expression level of E-cadherin was also decreased in the SSX2 over-expressing cells.

Over-expression of SSX2 prompts tumorigenesis in mice. To investigate the effect of SSX2 over-expression in vivo, we assessed tumorigenicity using SSX2 over-expressing MCF-7 and the control cells. After 8 weeks, 9/11 mice injected with SSX2 over-expressing MCF-7 showed tumors compared with 2/10 mice injected with the control cells (Fig. 7A and B). SSX2 over-expressing cells showed higher tumorigenesis in vivo. The tumor incidence was highly increased $(\mathrm{P}=0.009)$ in mice injected with SSX2 over-expressing MCF-7. As shown in Fig. 7C, analysis of H\&E-stained tissue sections confirmed that SSX2 over-expressing MCF-7 formed abundant tumors. We examined the expression of SSX2, ER $\alpha$ and EMT markers in the primary tumor tissues by immunohistochemistry. As expected, SSX2 was over expressed in the SSX2 xenograft sections, while ER $\alpha$ expression was down-regulated. The EMT related markers showed no changes (data not shown).

Identification of altered proteins in MCF-7 after over-expression of SSX2. Since SSX2 along with its family have an acidic tail and a KRAB domain which had some relationship with transcriptional repressor activity, proteomic methods were used to identify the altered proteins in MCF-7 after over-expression of SSX2 protein. 2-DE maps of SSX2 over-expressing MCF-7 and the control cells were constructed. After spot detection,
A

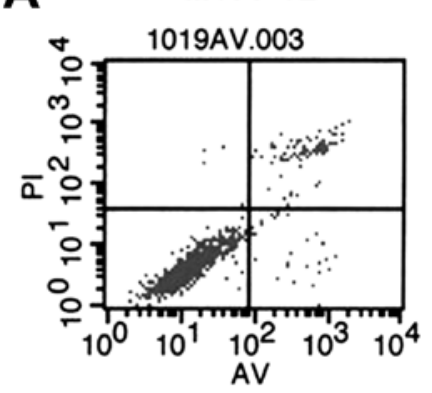

B

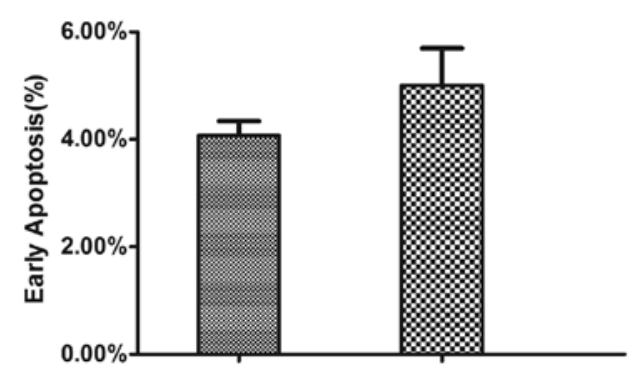

MCF7-C2-SSX2

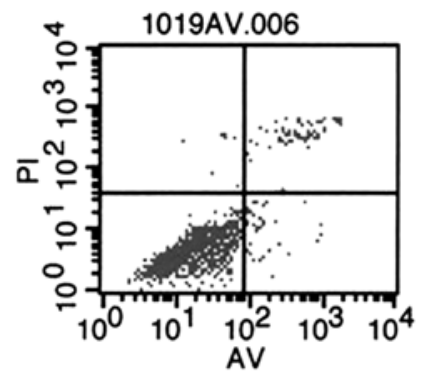

MCF7-C2

㘣 MCF7-C2-SSX2

Figure 4. Detection of apoptosis by flow cytometry in transfected cells. Compared with the control cells, the over-expression of SSX2 promoted a significant increase in the early-stage apoptosis rate of MCF-7. 


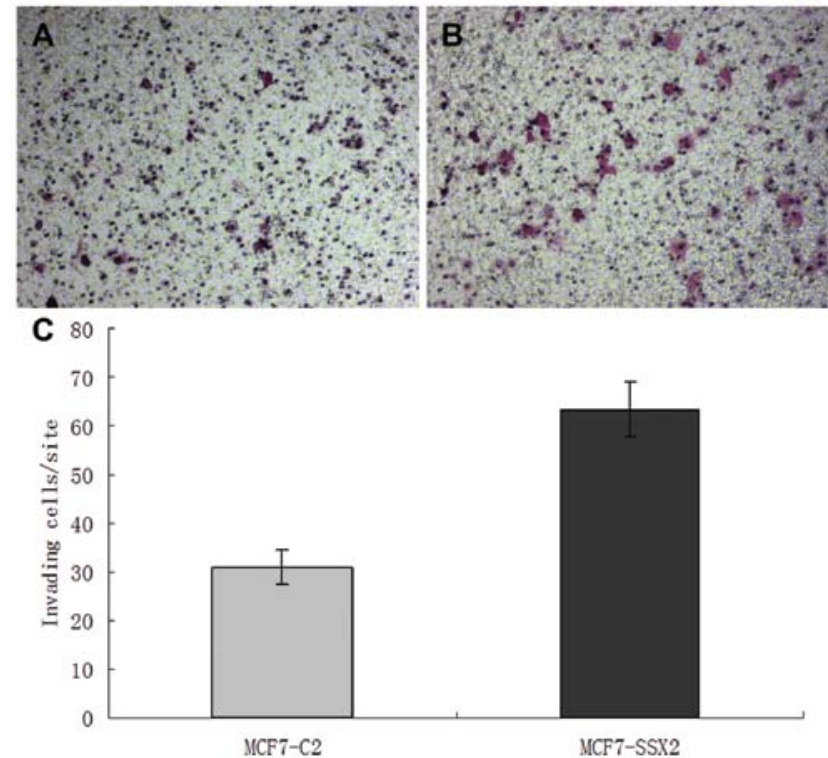

Figure 5. The invasiveness of SSX2 over-expressing MCF-7 and the control cells were assessed by transwell assays. The invasiveness through $8-\mu \mathrm{m}$ pore transwells was significantly $(\mathrm{P}<0.05)$ increased in SSX2 over-expressing MCF-7 compared with the controls. (A and B), original magnification x100.

23 protein spots were found markedly different in the SSX2 over-expressing MCF-7 compared with those in the control cells. Of all these protein spots, 10 spots increased and 13 spots

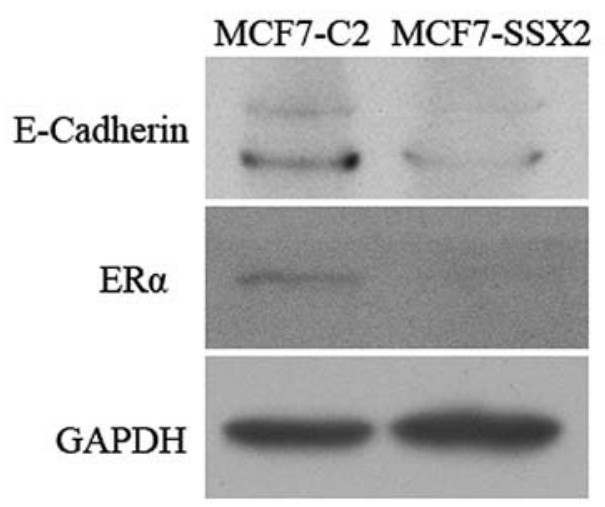

Figure 6. The expression level of ER $\alpha$ and E-cadherin was assessed after the transfection. As shown, both ER $\alpha$ and E-cadherin were markedly decreased in the SSX2 over-expressing MCF-7 compared with the control cells.

decreased after over-expression of SSX2 protein. Thirteen of these protein spots were identified successfully, corresponding to 11 known proteins. The identified protein spots are shown in the 2-DE maps (Fig. 8 and Table I), while the details of 11 known proteins are in Table II.

Validation of the 2-DE results by Western blot analysis. To validate the 2-DE results, we selected five representative proteins PDIA3, ARHGDIA, HSPB1, TAGLN, TUBB2C, for the detec-
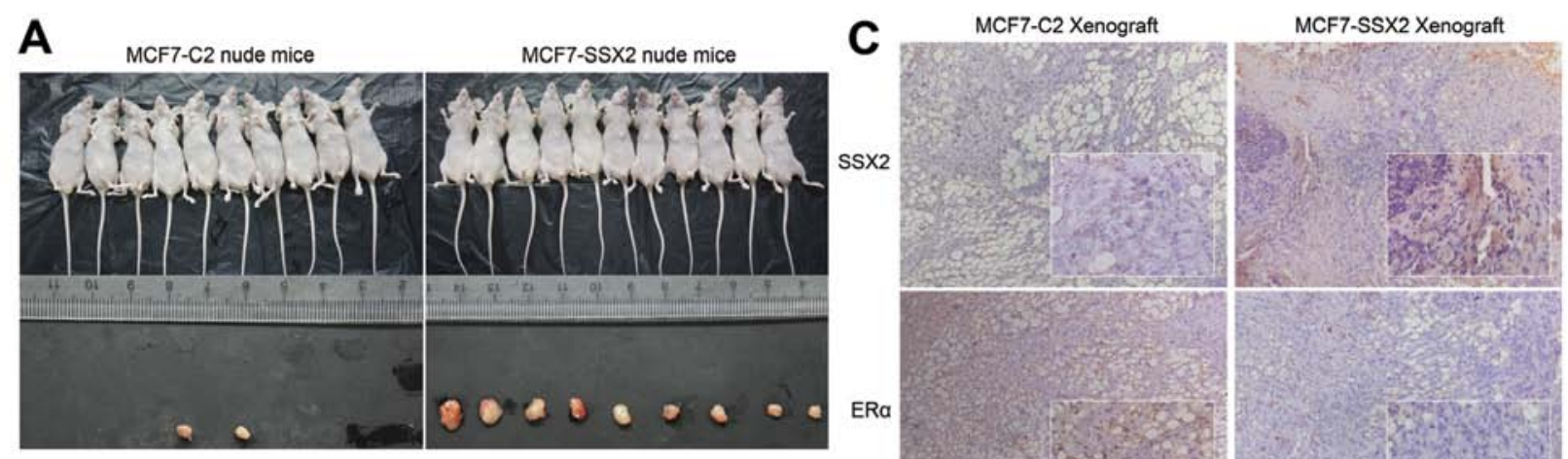

B
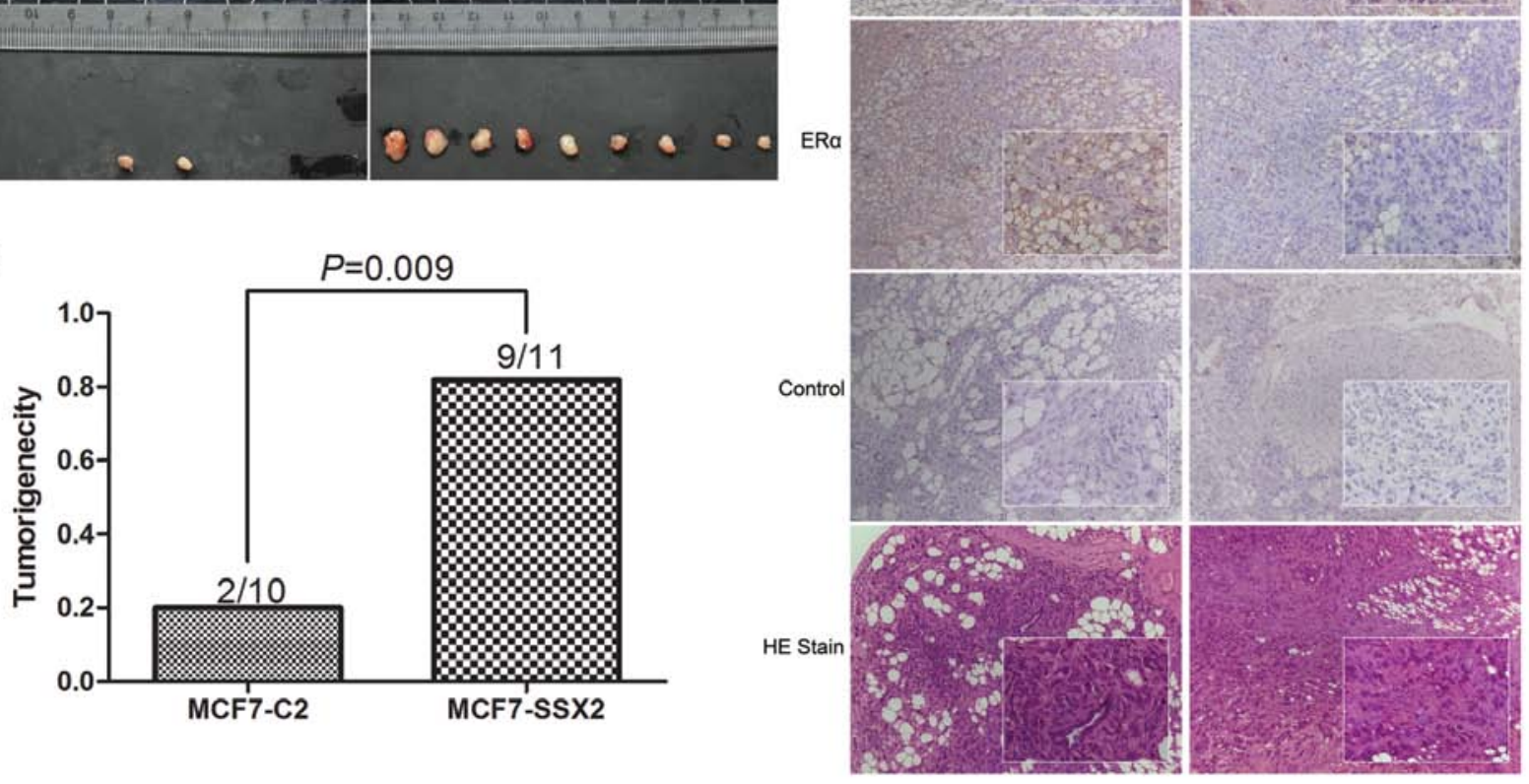

Figure 7. SSX2 over-expressing MCF-7 and control MCF-7 xenograft growth in nude mice. (A) Gross morphology of MCF-7 xenografts. (B) Histograms of tumorigenicity. In tumorigenicity assay, mice injected with SSX2 over-expressing cells had a significantly higher incidence $(\mathrm{P}=0.009)$ than the control cell injected mice. (C) Representative photomicrographs of anti-SSX2 and anti-ER $\alpha$ IHC performed on SSX2 over-expressing MCF-7 (right) and control MCF-7 (left) xenograft sections, original magnification x200 (the smaller figures, original magnification $\times 400$ ). 

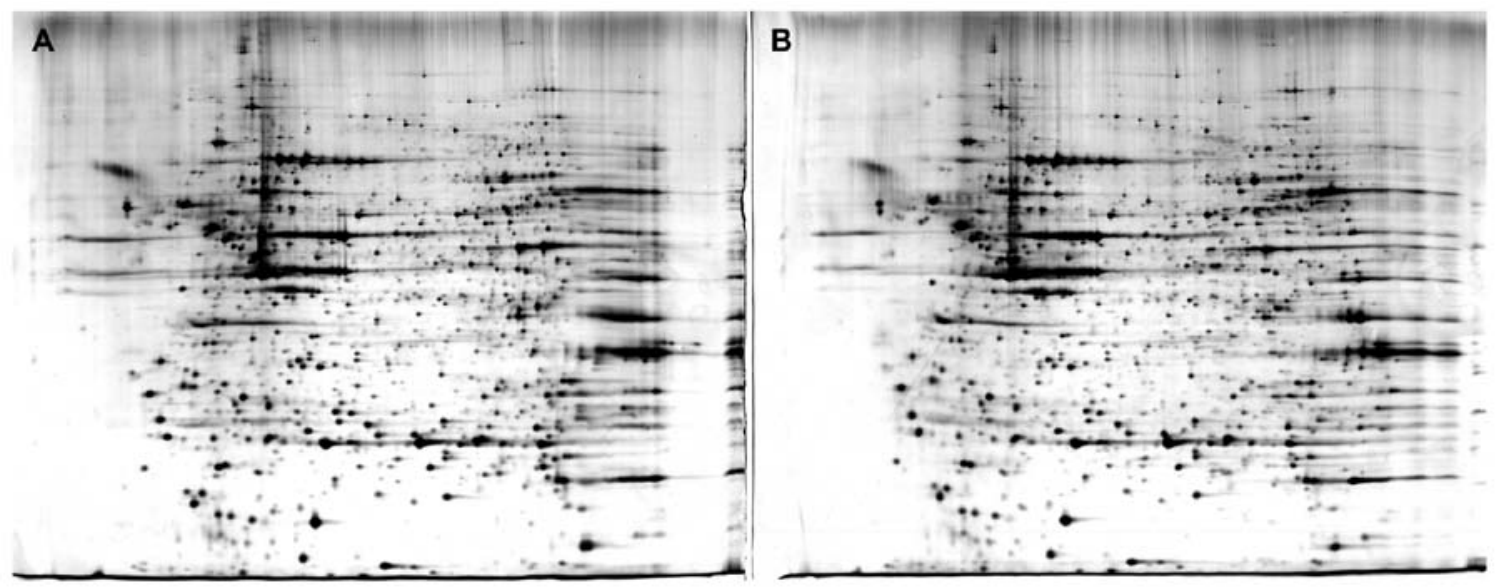

Figure 8. 2-DE gel images showing differential expression of the identified protein spots in the control MCF-7 (A) and SSX2 over-expressing MCF-7 (B).

A

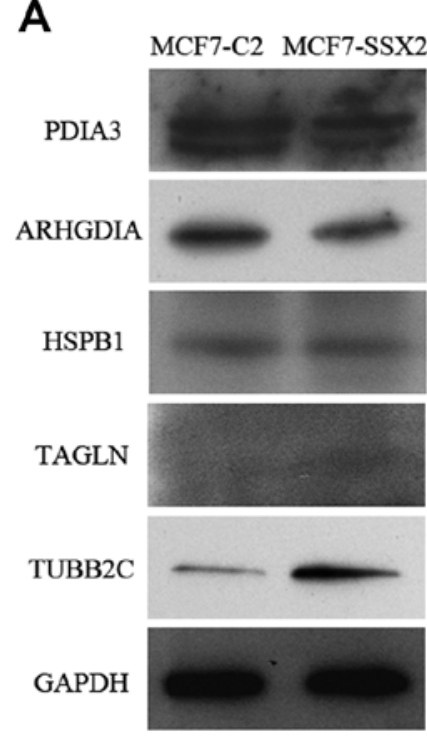

B

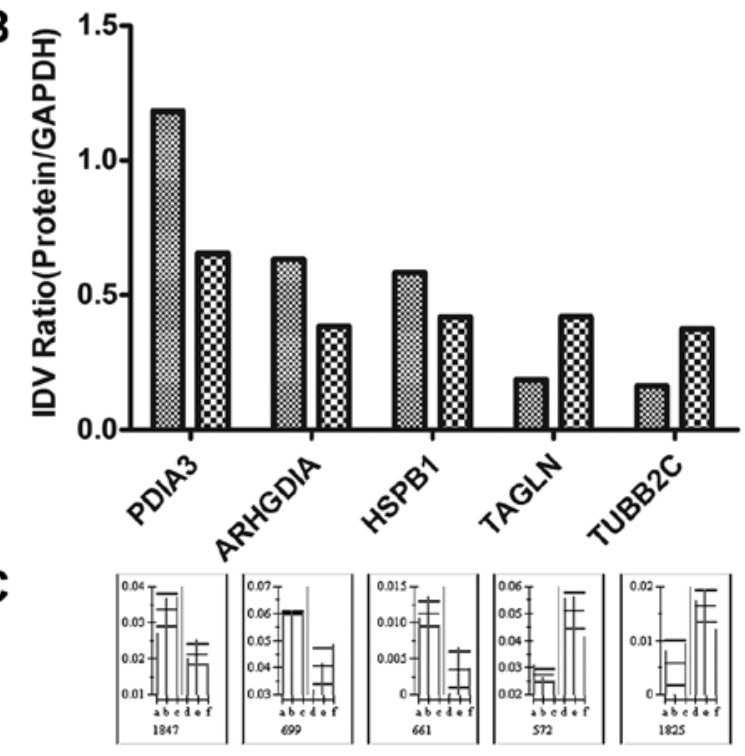

Figure 9. Western blot analysis was performed using anti-PDIA3, ARHGDIA, HSPB1, TAGLN, TUBB2C and GAPDH polyclonal antibodies for aliquots of total protein extracts prepared from the transfected cells (A). (B) Represented as the IDV ratio (protein/GAPDH). These results are in accordance with those determined using the ImageMaster 2D platinum software (C).

Table I. Identification of altered proteins between SSX2 overexpressing MCF-7 and the control MCF-7.

SSX2 over-expressing MCF-7/control

Increased Decreased

\begin{tabular}{lrr}
$\begin{array}{l}\text { Number of differentially expressed } \\
\text { protein spots }\end{array}$ & 11 & 14 \\
Number of identified protein spots & 10 & 13 \\
Number of identified proteins & 6 & 5 \\
\hline
\end{tabular}

tion of altered expression. As shown in Fig. 9, the expression levels of PDIA3, ARHGDIA, HSPB1 decreased in the SSX2 over-expressing MCF-7, while the levels of TAGLN, TUBB2C increased in the SSX2 over-expressing MCF-7, compared with the controls. These results are in accordance with those determined using the ImageMaster 2D platinum software.

\section{Discussion}

Emerging evidence has shown that auto-antibodies against CTA can be detected in primary breast cancer patients $(23,24)$. Previous studies have demonstrated that some CTA such as MAGE and NY-ESO-1 are expressed relatively high in triplenegative breast cancer which is of limited therapeutic options (11). The SSX protein family is a potential target applicable to many types of cancer instead of tumors of a restricted histological type (25). Owing to a high frequency of expression exclusively in cancer cells, CTA can become excellent cancer-specific antigen for breast cancer diagnosis and treatment.

SSX is found to be expressed in melanoma cells, fetal mesenchymal stem cells (MSCs), adult MSCs, and bone marrow. It was also observed that SSX expression is decreased 


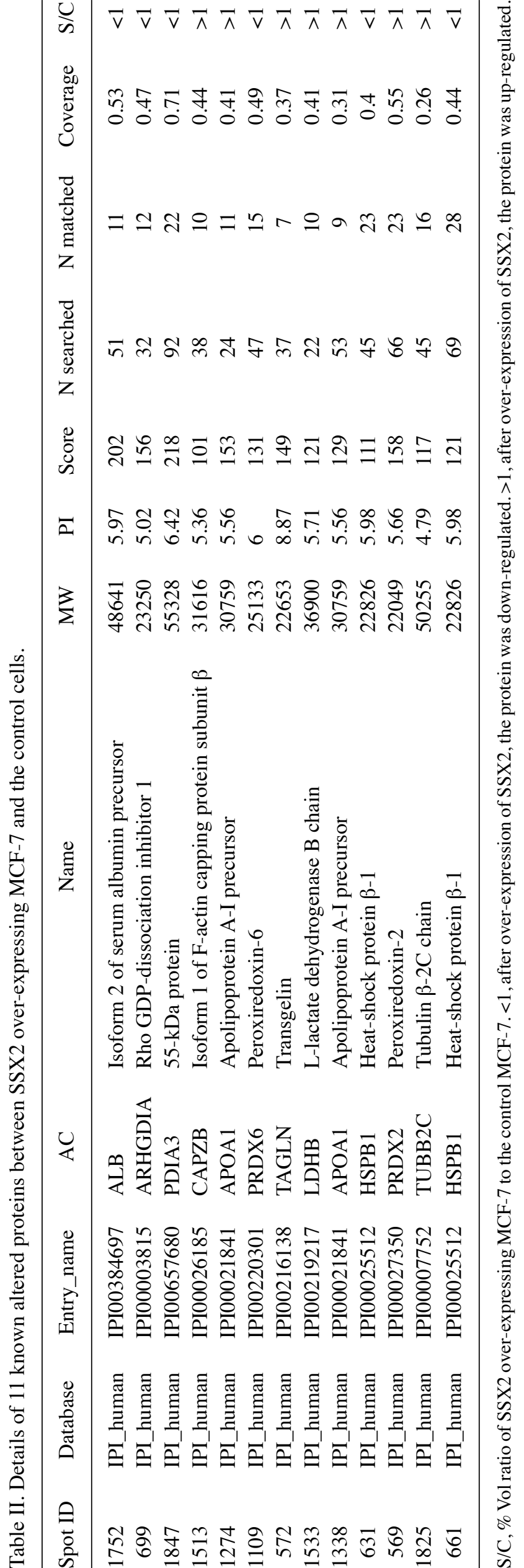

during MSC differentiation, implicating a link between SSX expression and the processes of self-renewal and tumorigenicity (26). In our study, most triple-negative breast cancer cell lines, particularly those with a mesenchymal phenotype (MDA-MB231, HCC1937, SUM1315), were SSX2-positive. It was implied that SSX2 was involved with invasive breast cancer cells. The differential expression between invasive and non-invasive cells adds the prerequisite for a broader application of antigen-specific immunotherapy.

Restricted expression of CTA in undifferentiated somatic and germ cells imply their essential role in embryonic development and tumor cell metastases (27). The SSX2 expression plasmid was designed and transfected into MCF-7 which is an ER $\alpha$-positive breast cancer cell line. Using the constructed stable transfected cells, we observed the effect of SSX2 on cell growth and invasion. We found that over-expression of SSX2 protein resulted in the decrease of cell proliferation and the increase of early apoptosis. Over-expression of SSX2 protein also enhanced the invasion of low invasive MCF-7 cells. These results are consistent with the differential mRNA expression between invasive and non-invasive breast cancer cell lines. Interestingly, $\mathrm{ER} \alpha$ and the epithelial marker E-cadherin expression were repressed in the SSX2 over-expressing MCF-7. However, there was no obvious change in the protein level of the mesenchymal markers such as vimentin (data not shown). MCF-7 cell tumors grow poorly without estradiol treatment in SCID mice $(28,29)$. Our results show that SSX2 favors the growth of MCF-7 xenografts since the growth rate of tumors was roughly four times higher for SSX2 over-expressing cells when compared with the controls (i.e., 81.8 vs. $20 \%$, respectively). Consistently, ER $\alpha$ expression was markedly down-regulated in SSX2 over-expressing MCF-7 xenografts. Previous studies verified that there is a strong positive correlation between ER $\alpha$ and E-cadherin expression in human breast cancer (30). In recent years, epithelial-mesenchymal transition (EMT) has been recognized as a key step during the progression of primary tumors into metastases as well as its original role during embryogenesis $(31,32)$. ER $\alpha$ signaling through Slug regulates E-cadherin and EMT (33). In our study, consistent decrease of both the epithelial marker and ER $\alpha$ suggested EMT features, which could explain the acquisitions of tumorigenesis and invasive capacity.

We performed a comparative proteomics study between SSX2 over-expressing MCF-7 and the control cells to elucidate the underlying molecular mechanisms. By comparing their peptide mass fingerprints to the IPI human database, eleven known proteins were identified and found to be a series of proteins related to proliferation, differentiation, apoptosis and cell survival (34-47). The SSX proteins are thought to act as transcriptional repressors through transcription repression domain (48). Tethering the KRAB domain to the promoter of ER target genes was able to cause suppression of gene expression (49). It has been demonstrated that binding the ligand-occupied ER $\alpha$ to estrogen response elements (EREs) in DNA, the receptor interacts with multiple interactive proteins to regulate transcription of target genes. ARHGDIA also known as Rho GDP dissociation inhibitor $\alpha$, is a negative regulator of the RhoGTPase family (40). It has been previously described to interact with $\mathrm{ER} \alpha$ and influence estrogen responsiveness (37). HSPB1 known as HSP27, interacts with ER and modulates ER function $(41,50)$. In 
addition, HSP27 has been recently shown to modulate p53 signaling and to suppress cellular senescence (38). In our study, decrease of ARHGDIA and HSPB1 was observed in SSX2 over-expressing MCF-7, which was consistent with down-regulation of ER $\alpha$ expression. There were several proteins related to invasion and migration. TAGLN (transgelin) is believed to be related to cell differentiation, cell migration, and the formation of podosomes and involved in tissue invasion and matrix remodeling $(34,35,39)$. It has been confirmed that TAGLN is able to promote migration and invasion of CSCs (42). Microtubules are cytoskeleton that play critical roles in cell division. Their subunit protein, tubulin, is a target for various antitumor drugs such as paclitaxel and docetaxel $(51,52)$. Alterations of $\beta$-tubulin isotypes in breast cancer cells may dictate, or predict, tumor cell behavior and responses to chemotherapeutic agents acting via the microtubule system (36). In our study, both TAGLN and TUBB2C expression were elevated in the SSX2 over-expressing group, which coincided with acquisition of invasiveness. Thus, the changing protein expression might contribute to those alternations observed after over-expression of SSX2 in MCF-7. Additional studies should help determine the potential molecular interaction between SSX2 and its target proteins.

In conclusion, we have shown for the first time that SSX2 expression was more pronounced in ER $\alpha$-negative breast cancer cells compared with the positive ones. The results of the present investigation suggest that SSX2 expression may enhance invasiveness in MCF-7 cells both in vivo and in vitro. The regulation of ER $\alpha$ signaling and EMT process by target proteins of SSX2 may explain the metastatic potential of breast cancer cells. It may be fundamentally important in understanding the mechanisms of SSX2 in tumor progression to prompt the exploitation of immunotherapeutic interventions especially for those patients with triple-negative breast cancer.

\section{Acknowledgements}

This work was supported in part by the National Natural Science Foundation of China (81071753 and 81172502), Wu Jie-Ping Foundation (320.670010009), the Six Kinds of Outstanding Talent Foundation of Jiangsu Province (2010-ws-079 to Wei He and 2009 to Qiang Ding), the Science and Education for Health Foundation of Jiangsu Province (RC2007054), the Natural Science Foundation of Jiangsu Province (BK2008476, BK2009438, BK2010581, BK2011853 and BK2011855), the Program for Development of Innovative Research Team in the First Affiliated Hospital of NJMU (IRT-008), and a project funded by the Priority Academic Program Development of Jiangsu higher Education Institutions (PAPD).

\section{References}

1. Jemal A, Siegel R, Xu J and Ward E: Cancer statistics, 2010. CA Cancer J Clin 60: 277-300, 2010.

2. Jager E, Jager D and Knuth A: Antigen-specific immunotherapy and cancer vaccines. Int J Cancer 106: 817-820, 2003.

3. Suri A: Cancer testis antigens - their importance in immunotherapy and in the early detection of cancer. Expert Opin Biol Ther 6: 379-389, 2006

4. Gure AO, Chua R, Williamson B, et al: Cancer-testis genes are coordinately expressed and are markers of poor outcome in non-small cell lung cancer. Clin Cancer Res 11: 8055-8062, 2005 .
5. Velazquez EF, Jungbluth AA, Yancovitz M, et al: Expression of the cancer/testis antigen NY-ESO-1 in primary and metastatic malignant melanoma (MM) - correlation with prognostic factors. Cancer Immun 7: 11, 2007.

6. Andrade VC, Vettore AL, Felix RS, et al: Prognostic impact of cancer/testis antigen expression in advanced stage multiple myeloma patients. Cancer Immun 8: 2, 2008.

7. Napoletano C, Bellati F, Tarquini E, et al: MAGE-A and NY-ESO-1 expression in cervical cancer: prognostic factors and effects of chemotherapy. Am J Obstet Gynecol 198: 99 e91-97, 2008.

8. Scanlan MJ, Gure AO, Jungbluth AA, Old LJ and Chen YT: Cancer/testis antigens: an expanding family of targets for cancer immunotherapy. Immunol Rev 188: 22-32, 2002.

9. Silina K, Zayakin P, Kalnina Z, et al: Sperm-associated antigens as targets for cancer immunotherapy: expression pattern and humoral immune response in cancer patients. J Immunother 34: 28-44, 2011.

10. Caballero OL and Chen YT: Cancer/testis (CT) antigens: potential targets for immunotherapy. Cancer Sci 100: 2014-2021, 2009.

11. Curigliano G, Viale G, Ghioni $\mathrm{M}$, et al: Cancer-testis antigen expression in triple-negative breast cancer. Ann Oncol 22: 98-103, 2011.

12. Jager D, Stockert E, Scanlan MJ, et al: Cancer-testis antigens and ING1 tumor suppressor gene product are breast cancer antigens: characterization of tissue-specific ING1 transcripts and a homologue gene. Cancer Res 59: 6197-6204, 1999.

13. Jager E, Karbach J, Gnjatic S, et al: Recombinant vaccinia/ fowlpox NY-ESO-1 vaccines induce both humoral and cellular NY-ESO-1-specific immune responses in cancer patients. Proc Natl Acad Sci USA 103: 14453-14458, 2006.

14. Marchand M, van Baren N, Weynants $P$, et al: Tumor regressions observed in patients with metastatic melanoma treated with an antigenic peptide encoded by gene MAGE-3 and presented by HLA-A1. Int J Cancer 80: 219-230, 1999.

15. Gure AO, Wei IJ, Old LJ and Chen YT: The SSX gene family: characterization of 9 complete genes. Int J Cancer 101: 448-453, 2002.

16. Dubovsky JA and McNeel DG: Inducible expression of a prostate cancer-testis antigen, SSX-2, following treatment with a DNA methylation inhibitor. Prostate 67: 1781-1790, 2007.

17. Naka N, Joyama S, Tsukamoto Y, et al: Quantification of SSX mRNA expression in human bone and soft tissue tumors using nucleic acid sequence-based amplification. J Mol Diagn 7: 187-197, 2005.

18. Tureci O, Sahin U, Schobert I, et al: The SSX-2 gene, which is involved in the $\mathrm{t}(\mathrm{X} ; 18)$ translocation of synovial sarcomas, codes for the human tumor antigen HOM-MEL-40. Cancer Res 56: 4766-4772, 1996.

19. Margolin JF, Friedman JR, Meyer WK, Vissing H, Thiesen HJ and Rauscher FJ III: Kruppel-associated boxes are potent transcriptional repression domains. Proc Natl Acad Sci USA 91: 4509-4513, 1994.

20. Witzgall R, O'Leary E, Leaf A, Onaldi D and Bonventre JV: The Kruppel-associated box-A (KRAB-A) domain of zinc finger proteins mediates transcriptional repression. Proc Natl Acad Sci USA 91: 4514-4518, 1994.

21. de Bruijn DR, dos Santos NR, Kater-Baats E, et al: The cancerrelated protein SSX2 interacts with the human homologue of a Ras-like GTPase interactor, RAB3IP, and a novel nuclear protein, SSX2IP. Genes Chromosomes Cancer 34: 285-298, 2002.

22. de Bruijn DR, van Dijk AH, Willemse MP and van Kessel AG: The $\mathrm{C}$ terminus of the synovial sarcoma-associated SSX proteins interacts with the LIM homeobox protein LHX4. Oncogene 27: 653-662, 2008

23. Mischo A, Kubuschok B, Ertan K, et al: Prospective study on the expression of cancer testis genes and antibody responses in 100 consecutive patients with primary breast cancer. Int J Cancer 118: 696-703, 2006.

24. Stockert E, Jager E, Chen YT, et al: A survey of the humoral immune response of cancer patients to a panel of human tumor antigens. J Exp Med 187: 1349-1354, 1998.

25. Smith HA and McNeel DG: The SSX family of cancer-testis antigens as target proteins for tumor therapy. Clin Dev Immunol 2010: 150591, 2010.

26. Cronwright G, Le Blanc K, Gotherstrom C, Darcy P, Ehnman M and Brodin B: Cancer/testis antigen expression in human mesechymal stem cells: down-regulation of SSX impairs cell migration and matrix metalloproteinase 2 expression. Cancer Res 65: 2207-2215, 2005. 
27. Ghafouri-Fard S and Modarressi MH: Cancer-testis antigens: potential targets for cancer immunotherapy. Arch Iran Med 12: 395-404, 2009.

28. Zhai YF, Esselman WJ, Oakley CS, Chang CC and Welsch CW: Growth of MCF-7 human breast carcinoma in severe combined immunodeficient mice: growth suppression by recombinant interleukin-2 treatment and role of lymphokine-activated killer cells. Cancer Immunol Immunother 35: 237-245, 1992.

29. Kubota T, Yamaguchi H, Watanabe M, et al: Growth of human tumor xenografts in nude mice and mice with severe combined immunodeficiency (SCID). Surg Today 23: 375-377, 1993.

30. Ye Y, Xiao Y, Wang W, Yearsley K, Gao JX and Barsky SH: ERalpha suppresses slug expression directly by transcriptional repression. Biochem J 416: 179-187, 2008.

31. Thiery JP and Sleeman JP: Complex networks orchestrate epithelial-mesenchymal transitions. Nat Rev Mol Cell Biol 7: 131-142, 2006.

32. Kang Y and Massague J: Epithelial-mesenchymal transitions: twist in development and metastasis. Cell 118: 277-279, 2004.

33. Ye Y, Xiao Y, Wang W, et al: ERalpha signaling through slug regulates E-cadherin and EMT. Oncogene 29: 1451-1462, 2010.

34. Lawson D, Harrison M and Shapland C: Fibroblast transgelin and smooth muscle SM22alpha are the same protein, the expression of which is down-regulated in many cell lines. Cell Motil Cytoskeleton 38: 250-257, 1997.

35. Shields JM, Rogers-Graham K and Der CJ: Loss of transgelin in breast and colon tumors and in RIE-1 cells by Ras deregulation of gene expression through Raf-independent pathways. J Biol Chem 277: 9790-9799, 2002

36. Shalli K, Brown I, Heys SD and Schofield AC: Alterations of beta-tubulin isotypes in breast cancer cells resistant to docetaxel. FASEB J 19: 1299-1301, 2005.

37. El Marzouk S, Schultz-Norton JR, Likhite VS, McLeod IX, Yates JR and Nardulli AM: Rho GDP dissociation inhibitor alpha interacts with estrogen receptor alpha and influences estrogen responsiveness. J Mol Endocrinol 39: 249-259, 2007.

38. O'Callaghan-Sunol C, Gabai VL and Sherman MY: Hsp27 modulates p53 signaling and suppresses cellular senescence. Cancer Res 67: 11779-11788, 2007.

39. Gimona M, Buccione R, Courtneidge SA and Linder S: Assembly and biological role of podosomes and invadopodia. Curr Opin Cell Biol 20: 235-241, 2008.
40. Boulter E, Garcia-Mata R, Guilluy C, et al: Regulation of Rho GTPase crosstalk, degradation and activity by RhoGDI1. Nat Cell Biol 12: 477-483, 2010.

41. Razandi M, Pedram A and Levin ER: Heat shock protein 27 is required for sex steroid receptor trafficking to and functioning at the plasma membrane. Mol Cell Biol 30: 3249-3261, 2010.

42. Dos Santos Hidalgo G, Meola J, Rosa ESJC, Paro de Paz CC and Ferriani RA: TAGLN expression is deregulated in endometriosis and may be involved in cell invasion, migration, and differentiation. Fertil Steril 96: 700-703, 2011.

43. Russell C: The role of albumin-bound Paclitaxel in metastatic breast cancer. Clin Adv Hematol Oncol 9: 469-471, 2011.

44. Lis CG, Grutsch JF, Vashi PG and Lammersfeld CA: Is serum albumin an independent predictor of survival in patients with breast cancer? J Parenter Enteral Nutr 27: 10-15, 2003.

45. Ivanenkov VV, Dimlich RV and Jamieson GA Jr: Interaction of S100a0 protein with the actin capping protein, CapZ: characterization of a putative S100a0 binding site in CapZ alpha-subunit. Biochem Biophys Res Commun 221: 46-50, 1996.

46. Soiland H, Soreide K, Janssen EA, Korner H, Baak JP and Soreide JA: Emerging concepts of apolipoprotein D with possible implications for breast cancer. Cell Oncol 29: 195-209, 2007.

47. Chang XZ, Li DQ, Hou YF, et al: Identification of the functional role of peroxiredoxin 6 in the progression of breast cancer. Breast Cancer Res 9: R76, 2007.

48. Lim FL, Soulez M, Koczan D, Thiesen HJ and Knight JC: A KRAB-related domain and a novel transcription repression domain in proteins encoded by SSX genes that are disrupted in human sarcomas. Oncogene 17: 2013-2018, 1998.

49. Ma ZQ, Tsai MJ and Tsai SY: Suppression of gene expression by tethering KRAB domain to promoter of ER target genes. J Steroid Biochem Mol Biol 69: 155-163, 1999.

50. Chen H, Hewison M and Adams JS: Control of estradiol-directed gene transactivation by an intracellular estrogen-binding protein and an estrogen response element-binding protein. Mol Endocrinol 22: 559-569, 2008.

51. Kennedy MJ, Donehower RC and Rowinsky EK: Treatment of metastatic breast cancer with combination paclitaxel/cyclophosphamide. Semin Oncol 22: 23-27, 1995.

52. Davidson NG: Single-agent paclitaxel at first relapse following adjuvant chemotherapy for breast cancer. Semin Oncol 22: 2-6, 1995. 